\title{
Visual Disorders in Neuroborreliosis: An Electro-physiological Investigation
}

\author{
Dan M. Psatta ${ }^{1}$ and Tatiana Rosca ${ }^{2}$ \\ 1. Center of Neurosciences of the Romanian Academy, Laboratory of Clinical Neurophysiology, Colentina Hospital, 8 Ezareni Street, \\ Bucharest 010258, Romania \\ 2. Neuro-ophthalmology Department, Saint Pantelimon Hospital, 59 Gala Galaction Street, Bucharest 011305, Romania
}

Received: January 11, 2015 / Accepted: January 19, 2015 / Published: January 30, 2015.

\begin{abstract}
Visual disorders may occur in Neuroborreliosis, Multiple Sclerosis, and many other diseases. The aim of this work was to find electro-physiological cues for differentiation. The study was based on the investigation of 178 patients presenting visual disorders. Out of them 57 were affected by Neuroborreliosis (demonstrated by positive serologic investigation: Western Blot and immuno-assay). The electro-physiological research consisted in Electroencephalography (EEG) Mapping and average Visual Evoked Potentials (VEPs) investigation. Characteristic for the Lyme disease was the non-homogenous slowing of the cortical electrical activity, associated with signs of excessive cortical excitability to flash stimulation. In Multiple Sclerosis (MS) there were no similar changes, but signs of sub-cortical white matter lesions. Primary cortical VEP components were significantly attenuated in amplitude in the Lyme disease and delayed in MS. Magnetic Resonance Imaging (MRI) showed the presence of not differentiated "demyelization lesions" in all these cases. The electro-physiologic investigation confirms the existence of cerebral (cortical) dysfunction in subjects serologically confirmed with Borrelia infection. It may be used in time for the follow up of the Lyme disease patients.
\end{abstract}

Key words: Neuroborreliosis, visual disorders, EEG Mapping, VEPs investigation.

\section{Introduction}

The diagnosis of Lyme disease is a difficult one. Characteristic symptoms such as facial paralysis, fatigue, light sensitivity, sleep disorders, depression do not occur in all the subjects presenting the Lyme disease [1-5]. Many of these symptoms are unspecific, and the disease may be misdiagnosed as Multiple Sclerosis, Chronique fatigue syndrome, Lupus, Fibromialgia (an autoimune or a degenerative disease). Visual disorders are also common in Multiple sclerosis, neuromyelitis and other viral encephalites [6]. Borreliosis was called the great imitator by some authors [7]. The purpose was to find new electro-physiologic markers for the differential diagnosis of this inflamatory encephalopathy.

Corresponding author: Dan M. Psatta, Ph.D. research fields: experimental and clinical neurophysiology. E-mail: dan.psatta@yahoo.com.

\section{Materials and Methods}

57 patients presenting a confirmed Borreliosis (by Western Blott and immunoassay) were included in the study (out of 178 patiets presenting visual disorders investigated). The electro-physiological research consisted in EEG Mapping and VEPs investigation.

The study of cerebral electrical activity was performed using an EEG investigation on 19 channels (the 10-20 International System), with left ear reference (electrodes impedance round $5 \mathrm{~K} \mathrm{Ohms).}$ Subjects were comfortably laid on a sofa, in a quiet environment. The EEG recording was performed first at rest, with the eyes closed, during $3 \mathrm{~min}$. In the second stage a similar EEG recording was performed during flash stimulation.

The results given in the clinical practice by the existing EEG Mapping technology are not satisfactory. Therefore the authors tried to elaborate a software of 
superior resolution, which the authors implemented actually on a commercially available Micromed EEG Mapping device $[8,9]$. The software package includes a "Source Derivation Filtering", based on an autoregressive model. From the electrical activity power of each source, calculated after Fourier analysis, the authors extract the weighted mean power of the 4-5 nearest electrical sources. Once the electrical interference excluded, one can find the true local EEG power magnitude. Experiments demonstrated the good resolution of this procedure and the fact that each source cumulates the surface cortical activity power and the deep electrical activity of the brain, projected by volume conduction on a radial vector. A supplementary facility of the program permits a full artifact rejection (power estimation on long 40-60 s artifact free EEG segments being made possible). The next step in the program consisted in power interpolation. With this, frequency band power Maps and various coefficients of power values can be plotted. Among the last, the authors preferably use the Delta-Theta/Alpha-Beta "abnormality ratio", paradoxically relevant also in case of EEG functional activation [9]. Other improvements: the use of standard (progressive) power scales, which made possible an easy comparison between different Maps, either transversally (between different subjects) or longitudinally (in the same subject, at various intervals of time), during medical treatment. Further, the authors implemented in the program a "functional" EEG Mapping procedure. Performing comparative EEG power evaluations (\%) in different conditions (stimulation, rest) one can observe and localize the effects of a given functional task on a regional EEG activity. In the study the author use it to assess the cerebral electrical activity changes induced by flash stimulation. The change in EEG power (cortical reactivity to light) is appreciated percent; the available reference scales on 11 steps measure reaction intensity. On these scales the percentage of $100 \%$ indicates the non reactive cortical areas. Increases in these percentages (colored in green, yellow, orange, red) indicate the enhancement in power of a given cerebral electrical activity (delta, theta, alpha, beta), or of a power ratio, in a given area during testing. This way, both the cortical damage and the changes in cerebral excitability that the authors expected to occur in Neuroborreliosis could be measured.

The examination was completed by ERG and Visual Evoked Potential comparative averaging [10], on time bases of $150 \mathrm{~ms}$ and $250 \mathrm{~ms}$.

\section{Results}

\subsection{Clinical Data}

Statistical data concerning symptoms are presented in Fig. 1. The incidence of the desease is almost equally distributed among ages, with a maximum between 30 and 40 years.

No pathological symptom seems to be pathognomonic for Borrelia. The high incidence of the visual disorders is due to the fact that patients came mainly from a neuro-ophtalmological departement. MRI was positive in $64 \%$ of cases, but it revealed unically white matter demyelinating lesions, which are common in MS and many other diseases. Fatigue was found in almost $60 \%$ of cases. Dizziness, paresthesiae, motor disorders are also common to MS. The authors found headaches in $28 \%$ of cases, muscular and articular pains in $22 \%$ and also various types of spells in $22 \%$. Fever or a tick bite were reported in only $10 \%$ of cases. Only $57 \%$ of the cases benefitted from the antibiotic therapy. Serologic examination (western blot and immuno assay) was positive in all the cases.

\subsection{EEG Mapping Results}

In normal subjects the partial power of Delta activity is higher frontally, but without exceeding $25 \%$. The Delta + Theta/Alpha + Beta ratio goes from 0.4 to 1.6. The cortical reaction to flash is restricted to the occipital lobe.

In all the cases in which the immunologic assay indicated a firm presence of Borerelia, the EEG Mapping 


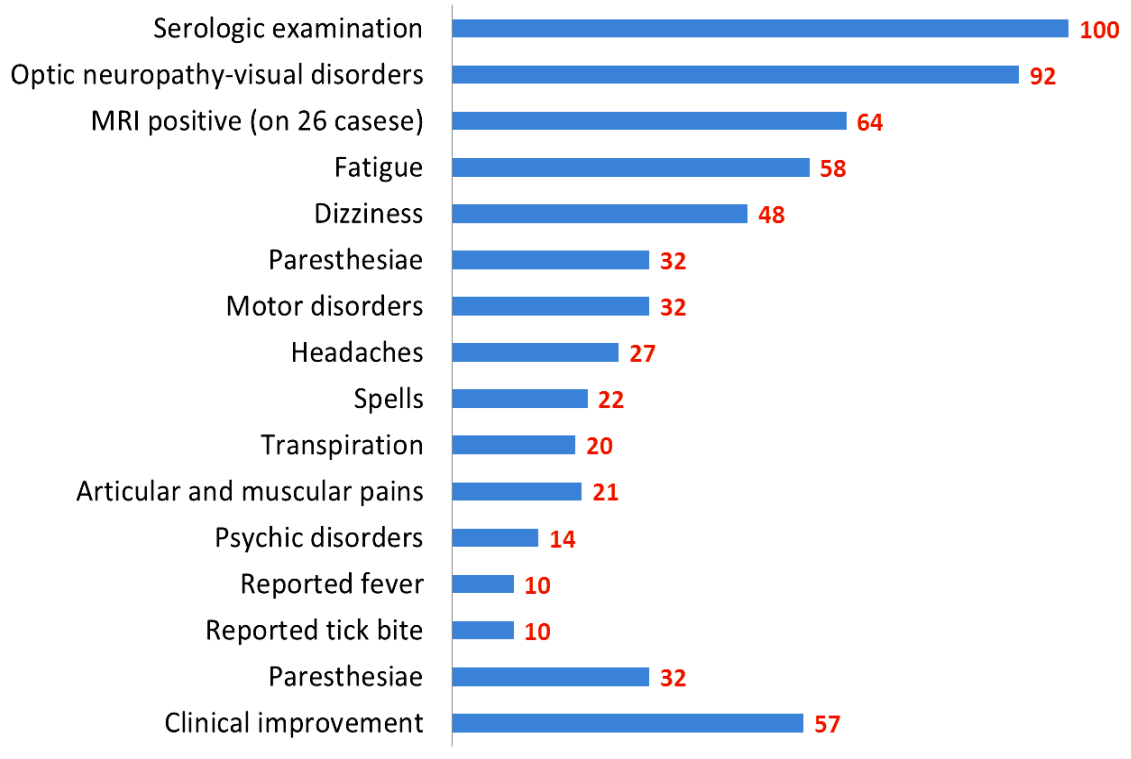

Fig. 1 Statistical analysis of clinical data (incidence \%).

images showed a non homogenous increase of slow waves (Delta or Theta) partial power. Any region of the brain could be involved preferentially, migration being possible in time. The Intensity of this enhancement was highly variable with the disease severity. However, the "abnormality" (Delta + Theta/Alpha + Beta) power ratio rarely exceeded a value of 4 (in severe encephalitis it can reach 10-15). A case is presented in Fig. 2, in comparison with images characteristic for normal cerebral activity and equivalent images from a case of Leuco-encephalitis. A diffuse abnormally high partial power Delta activity (up to $45 \%$ ), and a moderately enhanced power ratio (abnormal versus normal EEG activity) can be observed. On the functional Flash/Rest ratio investigation an abnormally high excitability of the right frontal neocortex can be observed. This hyper-excitability is characteristic, and can be also found in cases in which the electrical power at rest is only slightly disturbed. The functional Map showing the effect of binocular flash stimulation $(0.6$ Ws, $1 / \mathrm{sec}$ ) can be fully disorganized, with abnormal areas of deficit and of enhanced excitability.

In case of white matter lesions (Multiple Sclerosis, Vascular sub cortical Encephalopathy) the EEG abnormalities have a completely different pattern, evident in Fig. 2. Abnormalities at rest describe an arcade, occurring para-sagitally in the scalp projection area of "Centrum ovale" (the sub-cortical white matter). On a binocular flash stimulation, the same parasagital strips demonstrated a characteristically reduced intensity of the response.

\subsection{Visual Evoked Potentials Results}

Average VEPs (on 25 stimuli) recorded from both occipital lobes $(\mathrm{O} 1, \mathrm{O} 2)$ with $\mathrm{Fz}$ reference in one Normal, one Lyme and one Multiple Sclerosis representative subject, are presented in Fig. 3.

It was demonstrated previously $[11,12]$ that VEP is a composite response. It includes a N40 component (derived from the Geniculate Body), a N70/P100 component derived from the cortical occipital area, a N150 component (expressing reticular activation from the mid-line thalamic nuclei) and a N220-P300 component derived from the hippocampus. All these components are normal in the upper response presented in Fig. 3. The peak to peak amplitude of N70-P100 is above 10 micro volts normally.

In Neuroborreliosis in which visual disorders do occur (blurring, foggy vision, image distortions, colors neglect) the amplitude of VEP components is always 

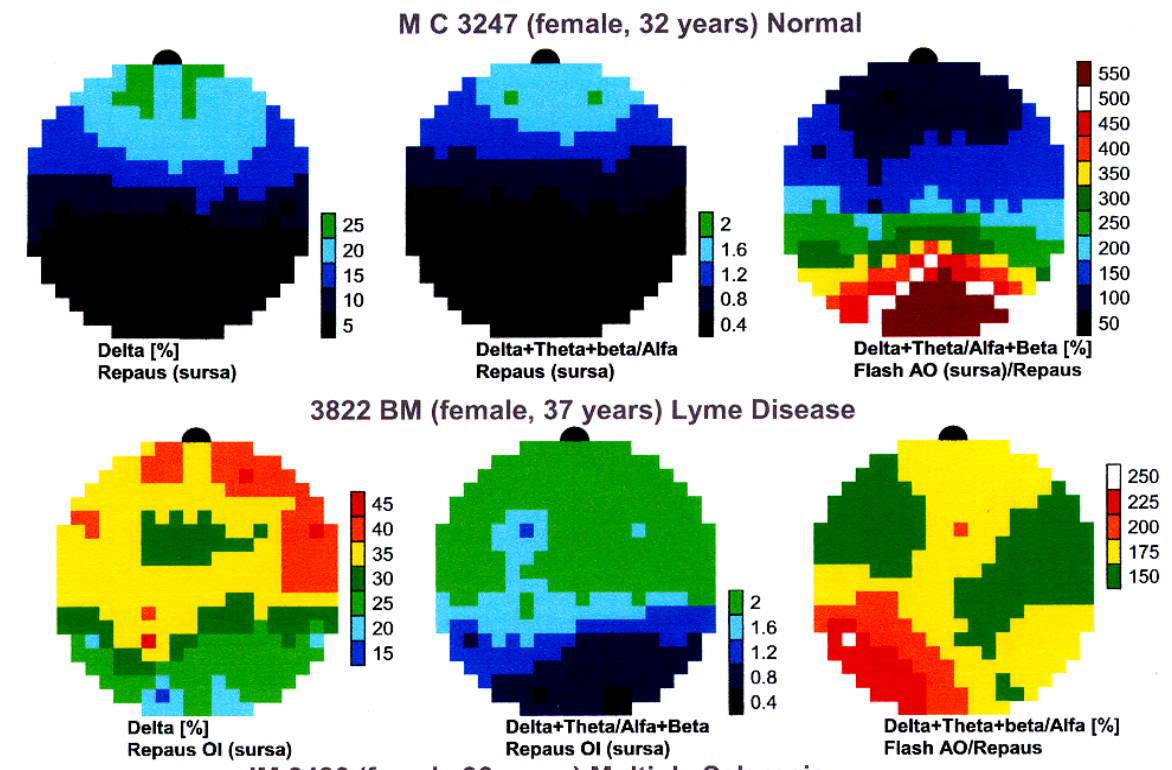

IM 3486 (female,36 years) Multiple Sclerosis
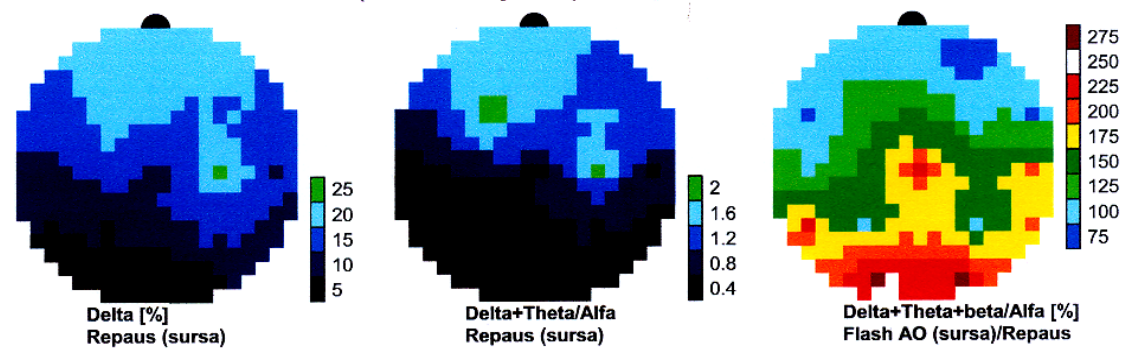

Fig. 2 EEG Mapping results in a normal (up), Lyme (middle) and a patient with Multiple Sclerosis (bottom). Rest mapping images (Delta \% and Power ratio) on the left, the response to Photic Stimulation on the right.

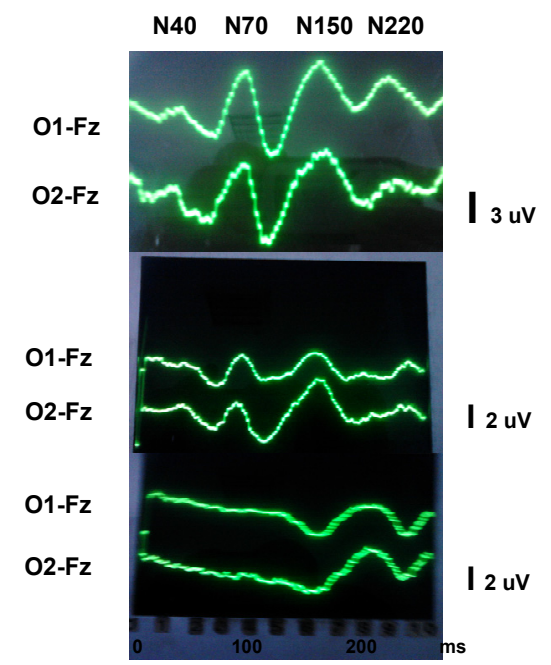

Fig. 3 Average Visual Evoked Potentials recorded in the two hemispheres. Time basis: $250 \mathrm{~ms}$; amplitude bars in micro volts. Up: normal subject; Middle: Lyme disease subject; Bottom: Subject affected by Multiple Sclerosis. Note the asymmetrically reduced amplitude and normal P100 latency in Lyme, and the enhanced P100 latency (160 ms) in Multiple Sclerosis. attenuated, N70/P100, amplitude being related to visual acuity. One can see in Fig. 3 that it can be also asymmetrically attenuated. The latency of P100 is normal instead (it does not exceed $125 \mathrm{~ms}$, the limit of normal latency).

In the case of Multiple Sclerosis one can observe both a severe attenuation in amplitude of primary VEP components, signaling axonal degeneration, and an enhanced P100 latency (160 ms), signaling a reduced conduction velocity (optic neuritis, peri-ventricular white matter sufferance).

\subsection{Antibiotic Therapy}

The patients came back to control every other year. The constantly utilized therapy, made according to ILADS, consisted in the association of antibiotic drugs from at least two classes: tetracyclines, cephalosporines, macrolides, fluoroquinolone and nitro-imidazole (in 


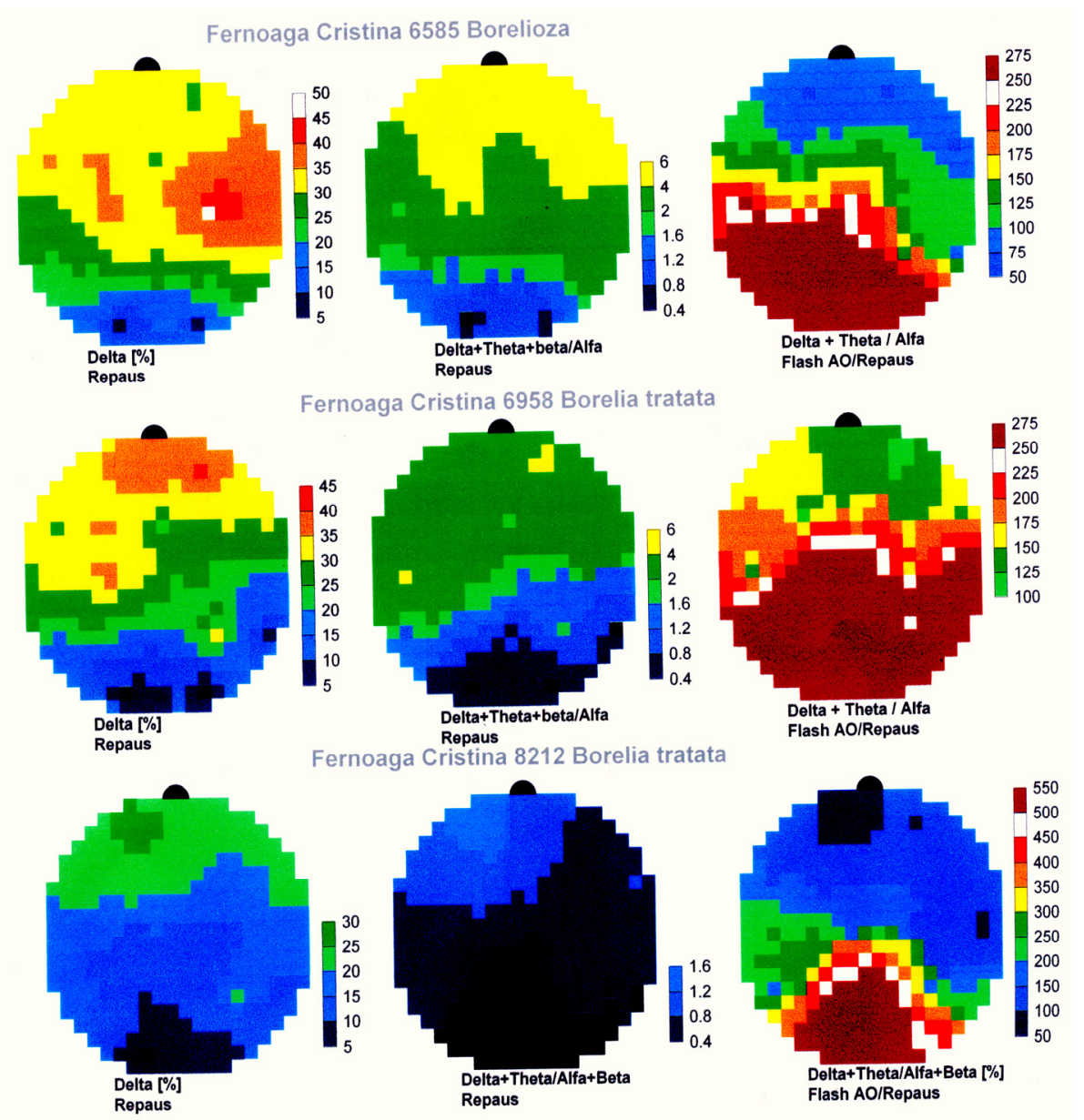

Fig. 4 Investigation during three years in a Borrelia infected patient. In the first row, map images before treatment. One can observe during the two years of treatment the progressive improvement of cerebral activity, both at rest (reduction of Delta power $\%$ and of the abnormality coefficient) and on bilateral flash stimulation (restriction of the response to the occipital area). Clinical and immunological parameters also improved.

five months periods). It was found that the results were extremely variable, from very good (Fig. 4) to moderate and even absent in $43 \%$ of the cases.

\section{Discussion}

Many authors discovered last time that serologic examination has not a full reliability. Wutte et al. [13] comparing the two ELISA's, immuno-blot and CXCL 13 testing, stated that only $26 \%$ of the patients were identified by all performed test methods. According to E Maloney [14], C6 Elisa sensitivity is $60 \%$ in the early and $73 \%$ in the late neuroborreliosis; IgG Western blot has a sensitivity of $72 \%$ in neuropatients. Seriburi et al. [15] found in revenge a high frequency of false positive IgM imunoblots for Borrelia burgdorferi in clinical practice $(27.5 \%$ of patients treated unnecessarily). Neuroborreliosis is considered confirmed when the immunoblot is stronger in the Cerebro-spinal Fluid than in the serum samples. All these authors mention the demand for new, supplementary methods of diagnosis.

The study showed that electro-physiological investigation may represent the required tool for the differential diagnosis in Lyme disease. The significant amount of visual disorders among the clinical manifestations of late stage Neuroborreliosis, which should be explained. Mapping images, with diffuse slow wave activity enhancement at rest, and areas of abnormal excitability by flash stimulation, suggest a certain cerebral involvement in Neuroborreliosis: a 
polio encephalitic type of cerebral damage (mainly cortical), contrasting those encountered in the leucoencephalitic sub-cortical type of diseases, like Multiple Sclerosis [16]. It was shown that spirochetes may induce the host cells to secrete products toxic to nerve cells: quinolinic acid and cytokines [17, 18]. Cellular destruction can fully explain the EEG Mapping abnormalities encountered.

ERG and VEPs investigation enhanced this information. In the Lyme disease the authors did not find the increased VEP latencies characteristic for demyelization, but a more or less reduced amplitude of the visual responses, encountered in case of axonal degeneration. MRI makes not this differentiation, sub-cortical demyelinating lesions being found in both disease entities.

Another subject of controversy in Neuroborreliosis is the antibiotic therapy. How long does the treatment be continued? It is expensive, unpleasant and of long duration. The follow up study revealed a very large variability of response to the antibiotic therapy among Lyme disease patients, from good or moderate to completely absent in $43 \%$ of cases. Relapses were possible. Lingering symptoms have also been described months to years after treatment. These ongoing symptoms could be related to an autoimmune response to the previous infection, or through the natural progression of disease states, when infection resolves much sooner than symptoms [19]. In the brain, Borrelia Burgdorferi induces the astrocytes to undergo astrogliosis [20], which could persist after the infection itself. In our opinion, a total lack of electro-physiologic response after two years of treatment, in spite of serologic normalization, should indicate an interruption of antibiotic therapy (its replacement by symptomatic medication).

\section{Acknowledgement}

The authors wish to thank to Drs. Cristi Moscu and Irina Butte (Romania) and Dr. Bela P. Boszik (Hungary) for the help in serologic investigation.

\section{References}

[1] Auwaerter, P. G., Aucutt, J., and Dumler, J. S. 2004. "Lyme Borreliosis: Molecular and Cellula Pathobiology and Prospects for Prevention, Diagnostic and Treatment." Expert Rev. Mol. Med. 6 (2): 1-22.

[2] Biesiada, G., Czepiel, J., Lesniack, M. R., Garlicki, A., and Mach, T. 2012. "Lyme Disease Review." Arch. Med. Sci. 8 (6): 978-82.

[3] Halperin, J. J. 2008. "Nervous System Lyme Disease." Infect. Dis. Clin. North. Am. 22 (2): 261-74

[4] Shadick, N. A., Philips, C. B., and Sangha, D. 1999. "Musculoskeletal and Neurologic Outcomes in Patients with Previously Treated Lyme Disease." Annals of Internal Medicine 131 (12): 919-26.

[5] Donta, S. T. 2014. "Lyme Disease: Chronic Fatigue and Fibromyalgia.” Chronic Disease International 1 (1): 1-2.

[6] Morrow, M. J., and Wingerchook, D. 2012. "Neuromyelitis optica." J . Neuroophtalmology 32: 154-66.

[7] Pachner, A. R. 1989. "Neurologic Manifestations of Lyme Disease; the New 'Great Imitator'." Rev. Infect. Dis. 6 (11): S1482-6.

[8] Psatta, D. M., Jipescu, I., and Matei, M. 1990. “A Personal Computer Used for Spectral Power EEG Mapping Based on Source Derivation." Rev. Roum. Neurol. Psychiat. 28: 79-86.

[9] Psatta, D. M., Olaru, M., and Matei, M. 1998. "Clinical Use of EEG Spectral Reaction Maps to Photic stimulation." Rom. J. Neurol. 36: 35-47.

[10] Psatta, D. M., Matei, M., and Grecu, P. I. 1989. "Simultaneous ERG and VEP Investigation in Ophtalmic Diseases: I. the Retinopathies." Neurologie et Psychiatrie 27 (1): 23-34.

[11] Psatta, D. M., and Matei, M. 1997. "Neural generators of Visual Evoked Potential components.” Rom. J. Neurol. 35: 13-37.

[12] Psatta, D. M., Olaru, M., and Matei, M. 2002. "Visual Inflow Travelling in the Brain Evidenced by Photic Evoked Potentials Mapping." Rom. J. Neurol. 40 (1/2): 27-44.

[13] Wutte, I. V., Arghelos, J., Crowe, B. A., Zenz, W., Daghofer, F., and Aberer, E. 2014. "Laboratory Diagnostic of Lyme Neuroborreliosis as Influenced by the Test Used: Comparison of the Two ELISAs, Immunoblot and of CXCL 13 Testing.” J. Neurol. Sci. 347 (1): 96-103.

[14] Maloney, E. 2010. "Insufficient Evidence and Poor Outcome: IDSA Treatment Recommendations Rightly Ignored". In: Philosophy, Etics and Humanities in Medicine.

[15] Seriburi, V., Ndukwe, N., Chang, T., Cox, R. E., and Wormser, G. P. 2012. "High Frequency of False Positive 
IgM Immunoblots for Borrelia Burgdorferi in Clinical Practice." Clin. Neurol. 18 (12): 1236-40.

[16] Psatta, D. M., and Matei, M. 2001. "Centrum Ovalae Demyelination Revealed by EEG Mapping in Multiple Sclerosis." Rom. J. Neurol. 39 (2): 85-97.

[17] Papanicolaou, D. A., Wilder, R. L., Manogagos, S. C., and Chrousos, G. P. 1998. "The Pathophysiological Role of Interleukin-6 in Human Disease." Annals of internal Medicine 128 (2): 127-37.

[18] Rasley, A., Amguita, J., and Mariott, I. 2002. "Borrelia
Burgdorferi Induces Inflamatory Mediator Production by Murine Microglia." J. Neuroimmunol. 130 (1): 22-31.

[19] Moore, K. S. 2015. "Lyme Diagnosis, Treatment Guide lines, and Controversy." J. for Nurse Practitioners 11 (1): 1-10.

[20] Ramesh, G., Alvarez, A. L., and Roberts, Z. D. 2003. "Pathogenesis of Lyme Neuroborreliosis: Borrelia Burgdorferi Lipoproteins Induce Both Proliferation and Apoptosis in Rhesus Monkey Astrocytes." Eur. J. of Immunology 33 (9): 2539-50. 